\title{
Recent astrophysical studies at the low-energy RI beam separator CRIB
}

\author{
H. Yamaguchi* \\ Center for Nuclear Study, Graduate School of Science, University of Tokyo \\ 2-1 Hirosawa, Wako, Saitama 351-0198, Japan \\ E-mail: yamag@ens.s.u-tokyo.ac.jp \\ D. Kahl, T. Nakao, \\ Center for Nuclear Study, Graduate School of Science, University of Tokyo \\ 2-1 Hirosawa, Wako, Saitama 351-0198, Japan \\ S. Kubono \\ RIKEN Nishina Center \\ 2-1 Hirosawa, Wako, Saitama 351-0198, Japan
}

CRIB (CNS Radioactive Ion Beam separator) is a low-energy RI beam separator at Center for Nuclear Study (CNS) of the University of Tokyo. Studies on proton and alpha resonance scatterings, $(\alpha, p)$ reactions, and other types of measurements ( $\beta$-decay lifetimes etc.) have been performed using RI beams at CRIB, motivated by interests on astrophysical reactions and exotic nuclear structure. Among the studies at CRIB, the measurement of ${ }^{7} \mathrm{Be}+\alpha$ resonant scattering is presented.

XII International Symposium on Nuclei in the Cosmos

August 5-12, 2012

Cairns, Australia

*Speaker. 


\section{Introduction}

CRIB [1,2] is a radio-isotope (RI) beam separator operated by Center for Nuclear Study (CNS), the University of Tokyo, installed in the RIBF facility of RIKEN Nishina Center. CRIB can produce low-energy $(<10 \mathrm{MeV} / \mathrm{u}) \mathrm{RI}$ beams in flight, using primary heavy-ion beams from the AVF cyclotron of RIKEN (K=70). Most of the RI beams are produced via direct reactions such as $(p, n),(d, p)$, and $\left({ }^{3} \mathrm{He}, n\right)$, taking place at an 8-cm-long gas target with a maximum pressure of 760 Torr. A cryogenic target system, in which the target gas can be cooled down to about $90 \mathrm{~K}$, is currently available, and an intense ${ }^{7} \mathrm{Be}$ beam of $2 \times 10^{8}$ pps was produced using the system [3]. Solid targets such as beryllium foil can also be used as the production target. The secondary beam is purified with an magnetic analysis using dipole magnets, and with a Wien filter, which can separate the beams according to their velocities. For relatively light RI beams such as ${ }^{7} \mathrm{Be}$, we can obtain a good purity of almost $100 \%$ after the Wien filter.

RI beams which have been produced at CRIB are listed in Table 1, with typical parameters. The RI-beam energy is optimized for each measurement, and can be changed according to the primary beam energy, the thickness of the production target, and other conditions. The intensity also varies depending on the conditions of the RI beam production.

\section{Nuclear Astrophysics Experiments}

The low-energy RI beams at CRIB are suitable for studies of astrophysical reactions. An experimental method often used was the thick-target method in inverse kinematics [4]. In that method, the beam energy is degraded in a thick reaction target, and reactions occur at various center-ofmass energies. We detect light particles emitted after reactions, and reconstruct the kinematics. This method has several advantages, namely, (a) we can perform simultaneous measurements of cross section of various excitation energies without varying the incoming RI beam energy, and (b) we can perform measurements at $180^{\circ}$ in center-of-mass angle, where the Coulomb scattering is minimal. Many fruitful results have been obtained at CRIB with this method [5-11].

A major topic of our interest is alpha-induced reactions. Several $(\alpha, p)$ reactions, such as ${ }^{14} \mathrm{O}(\alpha, p)[5],{ }^{11} \mathrm{C}(\alpha, p),{ }^{21} \mathrm{Na}(\alpha, p),{ }^{18} \mathrm{Ne}(\alpha, p)$, and ${ }^{30} \mathrm{~S}(\alpha, p)$ have been measured at CRIB. The elastic resonant scatterings of alpha particle using ${ }^{14} \mathrm{O},{ }^{21} \mathrm{Na},{ }^{7} \mathrm{Li}[11]$ and ${ }^{7} \mathrm{Be}$ beams have also been measured. These measurements are related to astrophysical $(\alpha, \gamma)$ reaction rates, and are relevant for studying nuclear cluster structure. Among the studies, the one on ${ }^{7} \mathrm{Be}+\alpha$ measurement, which is related to the cluster structure of ${ }^{11} \mathrm{C}$, is presented below.

The exotic cluster structures in the ${ }^{11} \mathrm{~B}$ and ${ }^{11} \mathrm{C}$ nuclei are attracting much attention in recent years [12]. The $3 / 2-$ state in ${ }^{11} \mathrm{~B}$ at the excitation energy $E_{\mathrm{ex}}=8.56 \mathrm{MeV}$ is regarded as a dilute cluster state [13], where two $\alpha$ particles and ${ }^{3} \mathrm{He}$ are weakly interacting. In particular, the alpha cluster structure in ${ }^{11} \mathrm{~B}$ was studied by measuring its isoscalar monopole and quadrupole strengths in the ${ }^{11} \mathrm{~B}\left(d, d^{\prime}\right)$ reaction, and the $8.56-\mathrm{MeV}$ state was suggested to have a dilute cluster structure $[14,15] .{ }^{11} \mathrm{C}$ is the mirror nucleus of ${ }^{11} \mathrm{~B}$, and such a cluster structure is also expected in it. A natural method to study the $\alpha$ cluster structure in ${ }^{11} \mathrm{C}$ is to form such a cluster state by the elastic scattering of ${ }^{7} \mathrm{Be}$ and $\alpha$. 
Table 1: RI beams produced at CRIB, with typical parameters of beam production. "Position" shows the name of the focal plane where the RI beam was measured. F2 is located before the Wien filter, and F3 is after that.

\begin{tabular}{|c|c|c|c|c|c|c|c|c|}
\hline \multicolumn{3}{|c|}{ PrimaryBeam } & \multirow[t]{2}{*}{ Target } & \multicolumn{5}{|c|}{ Secondary (RI) Beam } \\
\hline Ion & $\begin{array}{c}\text { Energy } \\
(\mathrm{MeV} / \mathrm{u})\end{array}$ & $\begin{array}{c}\text { Current } \\
(\mathrm{pnA})\end{array}$ & & Ion & $\begin{array}{l}\text { Energy } \\
(\mathrm{MeV} / \mathrm{u})\end{array}$ & $\begin{array}{c}\text { Intensity } \\
\text { (pps) }\end{array}$ & $\begin{array}{c}\text { purity } \\
(\%)\end{array}$ & Position \\
\hline${ }^{7} \mathrm{Li}$ & 5.0 & 500 & $\mathrm{H}_{2}$ & ${ }^{7} \mathrm{Be}$ & 3.8 & $5.0 \times 10^{6}$ & 100 & F3 \\
\hline${ }^{7} \mathrm{Li}$ & 5.6 & 1350 & $\mathrm{H}_{2}$ & ${ }^{7} \mathrm{Be}$ & 3.6 & $2.7 \times 10^{8}$ & 75 & $\mathrm{~F} 2$ \\
\hline${ }^{7} \mathrm{Li}$ & 6.5 & 333 & $\mathrm{D}_{2}$ & ${ }^{8} \mathrm{Li}$ & 5.0 & $2.0 \times 10^{5}$ & 100 & $\mathrm{~F} 3$ \\
\hline${ }^{6} \mathrm{Li}$ & 9.5 & 500 & ${ }^{3} \mathrm{He}$ & ${ }^{8} \mathrm{~B}$ & 5.2 & $1.0 \times 10^{4}$ & 30 & F3 \\
\hline${ }^{10} \mathrm{~B}$ & 7.8 & 200 & $\mathrm{CH}_{4}$ & ${ }^{10} \mathrm{C}$ & 6.1 & $1.6 \times 10^{5}$ & 90 & $\mathrm{~F} 2$ \\
\hline${ }^{11} \mathrm{~B}$ & 4.6 & 1000 & $\mathrm{H}_{2}$ & ${ }^{11} \mathrm{C}$ & 0.9 & $8.8 \times 10^{5}$ & 97 & F3 \\
\hline${ }^{10} \mathrm{~B}$ & 7.9 & 420 & ${ }^{3} \mathrm{He}$ & ${ }^{12} \mathrm{~N}$ & 5.8 & $6.0 \times 10^{2}$ & 30 & F3 \\
\hline${ }^{13} \mathrm{C}$ & 6.0 & 500 & $\mathrm{H}_{2}$ & ${ }^{13} \mathrm{~N}$ & 3.7 & $2.0 \times 10^{5}$ & 98 & F3 \\
\hline${ }^{18} \mathrm{O}$ & 6.1 & 200 & $\mathrm{Be}$ & ${ }^{14} \mathrm{C}$ & 6.2 & $2.8 \times 10^{5}$ & 19 & $\mathrm{~F} 2$ \\
\hline${ }^{14} \mathrm{~N}$ & 8.4 & 500 & $\mathrm{H}_{2}$ & ${ }^{14} \mathrm{O}$ & 2.4 & $5.0 \times 10^{5}$ & 93 & F3 \\
\hline${ }^{15} \mathrm{~N}$ & 5.0 & 0.1 & $\mathrm{D}_{2}$ & ${ }^{16} \mathrm{~N}$ & 0.9 & $2.5 \times 10^{1 \dagger}$ & 89 & $\mathrm{~F} 3$ \\
\hline${ }^{16} \mathrm{O}$ & 6.6 & 83 & $\mathrm{D}_{2}$ & ${ }^{17} \mathrm{~F}$ & 4.7 & $5.0 \times 10^{5}$ & 98 & $\mathrm{~F} 3$ \\
\hline${ }^{18} \mathrm{O}$ & 6.1 & 200 & $\mathrm{Be}$ & ${ }^{17} \mathrm{~N}$ & 5.6 & $3.5 \times 10^{5}$ & 24 & $\mathrm{~F} 2$ \\
\hline${ }^{16} \mathrm{O}$ & 10.4 & 86 & ${ }^{3} \mathrm{He}$ & ${ }^{17} \mathrm{Ne}$ & 4.9 & $2.4 \times 10^{2}$ & 0.3 & $\mathrm{~F} 2$ \\
\hline${ }^{18} \mathrm{O}$ & 6.1 & 200 & $\mathrm{Be}$ & ${ }^{17} \mathrm{O}$ & 5.4 & $1.2 \times 10^{5}$ & 8 & $\mathrm{~F} 2$ \\
\hline${ }^{18} \mathrm{O}$ & 5.9 & 35 & $\mathrm{H}_{2}$ & ${ }^{18} \mathrm{~F}$ & 3.2 & $6.2 \times 10^{5}$ & 73 & F3 \\
\hline${ }^{18} \mathrm{O}$ & 7.0 & 400 & $\mathrm{Be}$ & ${ }^{18} \mathrm{~N}$ & 5.7 & $3.4 \times 10^{5}$ & 6.1 & $\mathrm{~F} 2$ \\
\hline${ }^{16} \mathrm{O}$ & 6.8 & 560 & ${ }^{3} \mathrm{He}$ & ${ }^{18} \mathrm{Ne}$ & 3.7 & $5.1 \times 10^{5}$ & 81 & F3 \\
\hline${ }^{20} \mathrm{Ne}$ & 6.2 & 56 & $\mathrm{D}_{2}$ & ${ }^{21} \mathrm{Na}$ & 1.9 & $5.0 \times 10^{5}$ & 96 & F3 \\
\hline${ }^{20} \mathrm{Ne}$ & 6.1 & 100 & ${ }^{3} \mathrm{He}$ & ${ }^{22} \mathrm{Mg}$ & 3.7 & $3.5 \times 10^{3}$ & 18 & F3 \\
\hline${ }^{22} \mathrm{Ne}$ & 8.2 & 520 & $\mathrm{H}_{2}$ & ${ }^{22} \mathrm{Na}$ & 3.0 & $5.2 \times 10^{5}$ & 90 & F3 \\
\hline${ }^{24} \mathrm{Mg}$ & 7.4 & 60 & $\mathrm{D}_{2}$ & ${ }^{23} \mathrm{Mg}$ & 4.0 & $3.2 \times 10^{4}$ & 12 & $\mathrm{~F} 2$ \\
\hline${ }^{24} \mathrm{Mg}$ & 7.5 & 42 & $\mathrm{D}_{2}$ & ${ }^{25} \mathrm{Al}$ & 3.5 & $1.2 \times 10^{6}$ & 50 & F3 \\
\hline${ }^{24} \mathrm{Mg}$ & 7.5 & 200 & ${ }^{3} \mathrm{He}$ & ${ }^{26} \mathrm{Si}$ & 3.0 & $6.0 \times 10^{4}$ & 23 & F3 \\
\hline${ }^{28} \mathrm{Si}$ & 5.9 & 37 & $\mathrm{D}_{2}$ & ${ }^{30} \mathrm{P}$ & 1.0 & $2.6 \times 10^{3}$ & 30 & F3 \\
\hline${ }^{28} \mathrm{Si}$ & 7.3 & 77 & ${ }^{3} \mathrm{He}$ & ${ }^{30} \mathrm{~S}$ & 0.5 & $1.5 \times 10^{4}$ & 45 & F3 \\
\hline${ }^{40} \mathrm{Ar}$ & 4.5 & 118 & ${ }^{3} \mathrm{He}$ & ${ }^{39} \mathrm{Ar}$ & 3.7 & $7.0 \times 10^{2}$ & 4 & F3 \\
\hline${ }^{42} \mathrm{Ca}$ & 5.9 & 7 & ${ }^{3} \mathrm{He}$ & ${ }^{44} \mathrm{Ti}$ & 2.3 & $1.0 \times 10^{3} \neq$ & 15 & F3 \\
\hline${ }^{36} \mathrm{Ar}$ & 3.6 & 80 & ${ }^{12} \mathrm{C}$ & ${ }^{46} \mathrm{Cr}$ & 1.2 & $5.6 \times 10^{0}$ & 1.8 & F3 \\
\hline
\end{tabular}

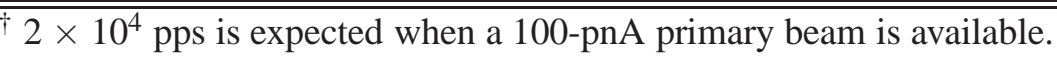

$\ddagger 1 \times 10^{4}$ pps is expected when a 70-pnA primary beam is available. 
The measurement of ${ }^{7} \mathrm{Be}+\alpha$ can be associated with the ${ }^{7} \mathrm{Be}(\alpha, \gamma)$ reaction. The ${ }^{7} \mathrm{Be}(\alpha, \gamma)$ reaction is considered to play an important role in the hot $p$ - $p$ chain and related reaction sequences [16]. Several reaction sequences including the ${ }^{7} \operatorname{Be}(\alpha, \gamma)$ reaction should take place in some hightemperature environments $\left(T_{9}>0.2\right)$. In the $v p$-process in core-collapse supernovae [17], the ${ }^{7} \operatorname{Be}(\alpha, \gamma)$ reaction may contribute as much as the triple- $\alpha$ process to the synthesis of elements heavier than boron at the relevant temperature of $T_{9}=1.5-3$, according to a theoretical calculation [18]. By our study, the resonant reaction rate should be evaluated more precisely by determining $\alpha$ widths for the resonances corresponding to such a high temperature region.

In 2010, we performed a measurement of ${ }^{7} \mathrm{Be}+\alpha$ resonant elastic and inelastic scatterings with the thick-target method in inverse kinematics at CRIB. A low energy ${ }^{7} \mathrm{Be}$ beam at $14.7 \mathrm{MeV}$ was produced using a $2.3-\mathrm{mg} / \mathrm{cm}^{2}$-thick hydrogen gas target and a primary ${ }^{7} \mathrm{Li}$ beam at $5.0 \mathrm{MeV} / \mathrm{u}$. The purity of the ${ }^{7} \mathrm{Be}$ beam was almost $100 \%$ after the Wien filter. The typical ${ }^{7} \mathrm{Be}$ beam intensity used in the measurement was $1-2 \times 10^{5}$ per second at the secondary target, and the main measurement using a thick helium-gas target was performed for 4 days, injecting $2.9 \times 10^{10}{ }^{7} \mathrm{Be}$ particles into the target.

\section{Beryllium $-7+\alpha$ results}

The analysis for the ${ }^{7} \mathrm{Be}+\alpha$ measurement is in progress. Here we present a preliminary analysis results for the excitation functions of the ${ }^{7} \mathrm{Be}+\alpha$ elastic and inelastic scatterings.

Inelastic scattering events producing ${ }^{7} \mathrm{Be}^{*}$ at the first excited state were identified by measuring 429-keV $\gamma$ rays with $\mathrm{NaI}$ detectors. We selected triple-coincidence events in which a ${ }^{7} \mathrm{Be}$ beam particle detected at the beam detector (MCP), an $\alpha$ or any other particle at the silicon detectors and a $\gamma$-ray at the NaI array. The $\gamma$-ray energy spectrum for those events is shown in Figure 1. The peak at $429 \mathrm{keV}$ was clearly identified, and small peaks around $511 \mathrm{keV}$ and $728 \mathrm{keV}$, which should be from positron annihilations and excited ${ }^{10} \mathrm{~B}$ produced via the ${ }^{7} \mathrm{Be}(\alpha, p){ }^{10} \mathrm{~B}^{*}$ reaction, were also observed.

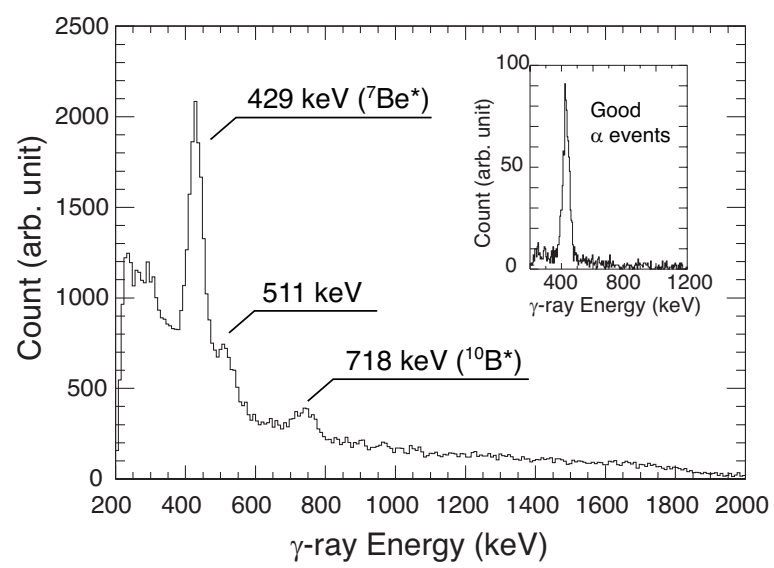

Figure 1: Energy spectrum of $\gamma$ rays for ${ }^{7} \mathrm{Be}-\alpha-\gamma$ triple-coincidence events. The insert shows the same spectrum for events with an $\alpha$ particle identified, after a beam position selection.

The excitation functions of the elastic and inelastic scatterings are shown in Figure 2. No 


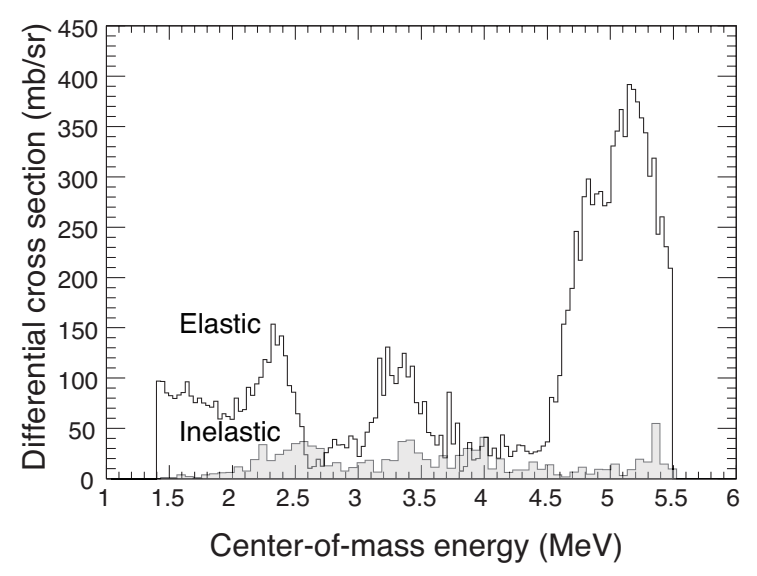

Figure 2: Excitation functions of ${ }^{7} \mathrm{Be}+\alpha$ elastic and inelastic (shaded spectrum) scattering cross sections.

previous measurements to be directly compared with these excitation functions are known. In the present excitation function of the elastic scattering, a structure having several peaks, which should correspond to the resonance structure in ${ }^{11} \mathrm{C}$, was observed. We expect that the parameters of these resonances could be determined by performing an R-matrix analysis.

\section{Summary}

CRIB is a facility which can produce low-energy RI beams with good purity and intensity. Many astrophysical reactions have been studied at CRIB, using its low-energy RI beams. We performed a measurement of ${ }^{7} \mathrm{Be}+\alpha$ elastic resonance scattering using the thick-target method in inverse kinematics for the first time. The resonance parameters to be determined in this study, such as the spin, parity and alpha width, related with the spectroscopic factor of the $\alpha$-cluster configuration, would provide valuable information for the $\alpha$-cluster structure in the high excited states, and the astrophysical ${ }^{7} \mathrm{Be}(\alpha, \gamma)$ reaction rates in high-temperature phenomena.

The experiment was performed at RI Beam Factory operated by RIKEN Nishina Center and CNS, the University of Tokyo. We are grateful to the RIKEN and CNS accelerator staff for their help. This work was partly supported by JSPS KAKENHI (No. 21340053) from the Ministry of Education, Culture, Sports, Science and Technology (MEXT) of Japan.

\section{References}

[1] S. Kubono et al., Eur. Phys. J. A13 (2002) 217.

[2] Y. Yanagisawa et al., Nucl. Instrum. Meth. Phys. Res., Sect. A 539 (2005) 74-83.

[3] H. Yamaguchi et al., Nucl. Instrum. Meth. Phys. Res., Sect. A 589 (2008) 150-156.

[4] K. P. Artemov et al., Sov. J. Nucl. Phys 52 (1990) 408.

[5] M. Notani et al., Nucl. Phys. A 746 (2004) 113c-117c.

[6] T. Teranishi et al., Phys. Lett. B 556 (2003) 27-32. 
[7] T. Teranishi et al., Phys. Lett. B 650 (2007) 129-134.

[8] J. J. He et al., Phys. Rev. C 76 (2007) 055802.

[9] H. Yamaguchi et al., Phys. Lett. B 672 (2009) 230-234.

[10] J. J. He et al., Phys. Rev. C 80 (2009) 015801.

[11] H. Yamaguchi et al., Phys. Rev. C 83 (2011) 034306.

[12] Y. Kanada-En'yo, Phys. Rev. C 75 (2007) 024302.

[13] A. Tohsaki, H. Horiuchi, P. Schuck, and G. Röpke, Phys. Rev. Lett. 87 (2001) 192501.

[14] T. Kawabata et al., Phys. Rev. C 70 (2004) 034318.

[15] T. Kawabata et al., Phys. Lett. B 646 (2007) 6-11.

[16] M. Wiescher, J. Görres, S. Graff, L. Buchmann, and F.-K. Thielemann, Astrophys. J. 343, 352-364 (1989).

[17] C. Fröhlich, P. Hauser, M. Liebendörfer, G. Martínez-Pinedo, F.-K. Thielemann, E. Bravo, N. Zinner, W. Hix, K. Langanke, A. Mezzacappa, and K. Nomoto, Astrophys. J. 637, 415 (2006).

[18] S. Wanajo, H.-T. Janka, and S. Kubono, Astrophys. J. 729, 46 (2011).

[19] F. Ajzenberg-Selove, Nucl. Phys. A 506 (1990) 1. 